\title{
Lesser Curvature of the Stomach
}

National Cancer Institute

\section{Source}

National Cancer Institute. Lesser Curvature of the Stomach. NCI Thesaurus. Code C12261.

The right border of the stomach. It is concave in shape and extends from the cardiac orifice to the pyloric orifice. 